\title{
Range measurement by a digital camera using flash
}

\author{
Naoya Ogawa*a ${ }^{* a}$ Kazunori Umeda ${ }^{\mathrm{b}}$ \\ ${ }^{a}$ Course of Precision Eng., Chuo Univ., 1-13-27 Kasuga, Bunkyo-ku, Tokyo 112-8551, Japan; \\ ${ }^{\mathrm{b}}$ Dept. Presicion Mechanics, Chuo Univ., 1-13-27 Kasuga, Bunkyo-ku, Tokyo 112-8551, Japan
}

\begin{abstract}
Various methods have been proposed until now for range measurement or three dimensional shape reconstruction. However, most of them need a large-scale equipment or a special environment. This paper proposes a technique which obtains a range image easily under a general environment using only an off-the-shelf digital camera. Distance is calculated by obtaining the irradiance of scene lighted by the flash of a digital camera using the fact that the intensity of reflected light of the flash is inversely proportional to the square of the distance from the object. The irradiance is obtained by subtracting an image without the flash from an image with the flash. The image without the flash is used to obtain the reflectance ratio at each pixel. The intensity of reflected light of the flash is affected by the inclination of the object surface. A method to estimate the inclination at each pixel is proposed which uses the change of the irradiance in adjacent pixels. The inclination is formulated as the function of the rate of change, and thus the inclination can be calculated by the rate which is easily obtained from the image. Additionally, color information is simultaneously obtained because visible light is used. Assumptions in the method are that the object surface has no specular reflection and the flash is set at the same position as the center of the lens. Experiments show that a range image is roughly obtained by the proposed method, and furthermore, that proper distance is obtained for inclined surfaces.
\end{abstract}

Keywords: digital camera, flash, range image, range measurement, diffuse surface, reflectance ratio, inclination of surface

\section{INTRODUCTION}

Various methods have been proposed until now for range measurement or three dimensional shape reconstruction. ${ }^{1-3}$ Stereo vision is well-known and fundamental for many other methods. It measures distance by triangulation with two or more cameras. There is a commercially available system. ${ }^{4}$ However, the correspondence problem is a big problem. To solve this problem, many active methods have been proposed, such as laser scanners based on triangulation ${ }^{5}$ or time-of-flight principles, the light section method, the photometric stereo ${ }^{6}$ etc. However, most of them need a large-scale equipment or a special environment. Many laser scanners are large, and to realize the photometric stereo, controlled illumination in a dark room is necessary. Special equipments to achieve three dimensional reconstruction are presented such as CyberModeler. ${ }^{7}$ However, this needs a turntable and a background paper.

A handy and inexpensive system is presented called Motion Processor ${ }^{8}$ for clipping an object from back ground and reconstructing a rough three dimensional shape. This system has a CCD camera and near-infrared LEDs around the CCD camera. The light from LEDs reflects on the object surface and reaches to the CCD. The intensity of the reflected light on the CCD becomes weak in proportion to the square of the distance to the object. Using this characteristic, the three dimensional shape can be reconstructed roughly. Referring to this technique, we propose a method for range measurement with a digital camera by using the flash. With this method, range and color information can be obtained easily under a general environment with the visible light.

*ogawa@sensor.mech.chuo-u.ac.jp, http://www.mech.chuo-u.ac.jp/umedalab/index_e.html

Optomechatronic Machine Vision, edited by Kazuhiko Sumi, Proceedings of SPIE Vol. 6051, 605108, (2005) · 0277-786X/05/\$15 · doi: 10.1117/12.647730

Proc. of SPIE Vol. $6051605108-1$ 


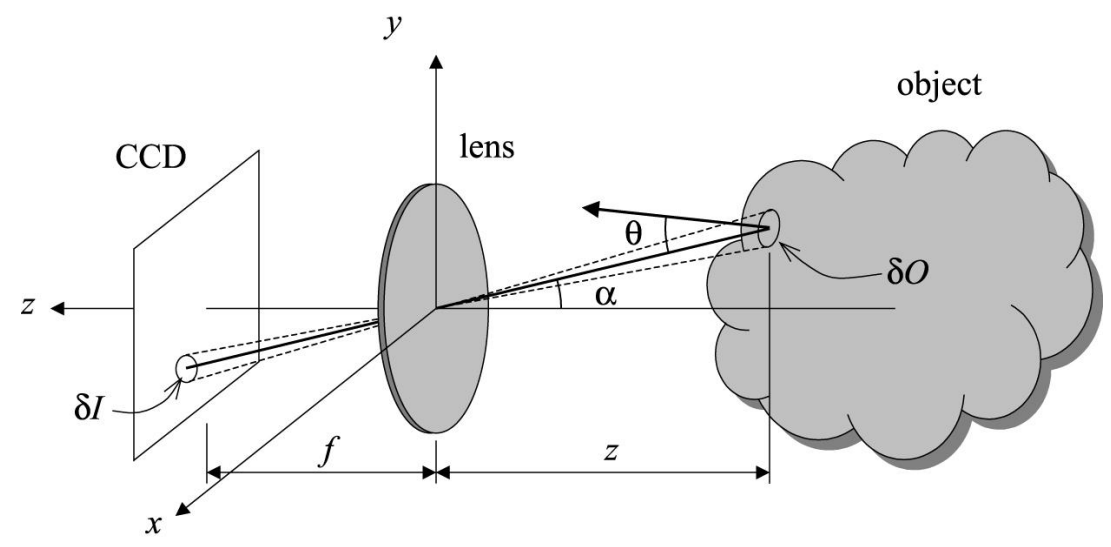

Figure 1. Parameters for range measurement

\section{METHOD OF RANGE MEASUREMENT}

\subsection{Outline of the method}

Range information is obtained from two images by this method, images with and without the flash.

Pixel value of the image is proportional to the irradiance on the CCD. Since irradiance has linearity, the subtraction of the irradiance of the image without the flash from that with the flash becomes the irradiance of the image with only the flash. Range information can be calculated since the intensity of the reflected flash light is inverse-proportional to the square of the distance to the object. It is not necessary to prepare a special environment since this method does not need to know the illumination environment other than the flash. So, the range image can be obtained easily and inexpensively with this method.

\subsection{Equation for calculating the distance}

In Fig. 1, the relation between the radiance of a certain area on the object and the irradiance of the area on the CCD corresponding to the area of the object is as follows. Let us set $\delta O$ is the small area on the object surface, $\delta I$ is the small area on the CCD corresponding to $\delta O, \alpha$ is the angle made by the optical axis and the light which goes to the center of the lens from $\delta O$, and $\theta$ is the angle made by the normal of the object surface and the light described above. The irradiance of $\delta I$ is given by the following equation. ${ }^{6}$

$$
E_{C C D}=L_{\text {object }} \frac{\pi}{4}\left(\frac{D}{f}\right)^{2} \cos ^{4} \alpha
$$

where $L_{\text {object }}$ is the radiance of $\delta O$. By introducing the Bidirectional Reflectance Distribution Function (BRDF) $\rho\left(\theta_{i}, \phi_{i} ; \theta_{e}, \phi_{e}\right)$, eq. (1) becomes

$$
E_{C C D}=\rho\left(\theta_{i}, \phi_{i} ; \theta_{e}, \phi_{e}\right) E_{\text {object }} \frac{\pi}{4}\left(\frac{D}{f}\right)^{2} \cos ^{4} \alpha
$$

where $E_{\text {object }}$ is the irradiance of $\delta O$. The BRDF is the function showing the characteristic of the reflection property of the surface in the light source direction $\left(\theta_{i}, \phi_{i}\right)$ and the viewing direction $\left(\theta_{e}, \phi_{e}\right)$. In other words, it is the function showing the ratio of the radiance to the irradiance. Now, we consider the case that the light source is only the flash. The irradiance of $\delta O$ is then given by

$$
E_{\text {object }}=\frac{I \delta \omega}{\delta O} \cos \theta_{f}
$$

where $I$ is the radiant intensity of the flash, $\theta_{f}$ is the angle made by the light to the $\delta O$ from the flash and the normal of the object surface and $\delta \omega$ is the solid angle seeing the small area of the object surface from the flash. 


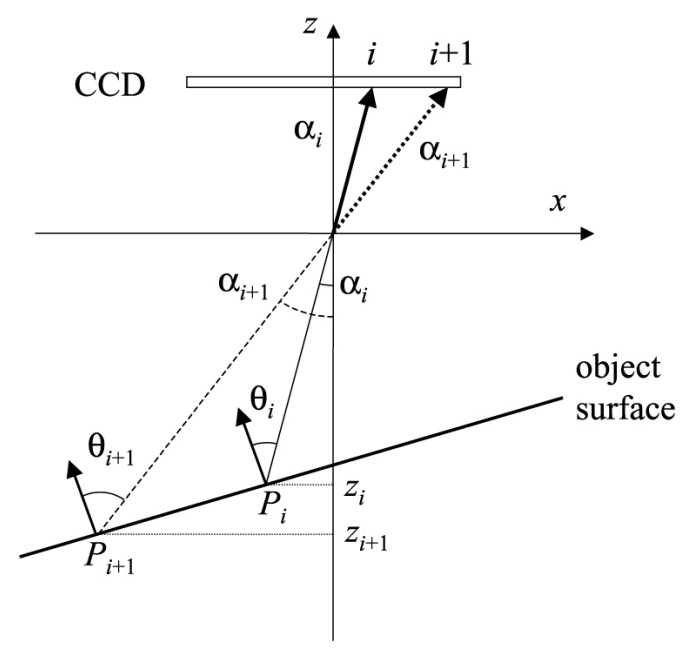

Figure 2. Adjacent points of tilted surface

$\delta \omega$ is given by

$$
\delta \omega=\frac{\delta O \cos \theta_{f}}{\left(z / \cos \alpha_{f}\right)^{2}}
$$

where $\alpha_{f}$ is the angle made by the light to $\delta O$ and the optical axis. When eq. (3), (4) are taken into consideration, eq. (2) becomes

$$
E_{C C D}=\rho\left(\theta_{f}, \phi_{f} ; \theta, \phi\right) I \frac{1}{z^{2}} \cos ^{2} \alpha_{f} \cos ^{2} \theta_{f} \frac{\pi}{4}\left(\frac{D}{f}\right)^{2} \cos ^{4} \alpha
$$

Let us set the irradiance of the CCD with and without the flash $E_{C C D}^{F}$ and $E_{C C D}^{N F}$ respectively. Then $E_{C C D}=$ $E_{C C D}^{F}-E_{C C D}^{N F}$.

Let us assume that the position of the flash is same as that of the center of the lens. Then $\alpha_{f}=\alpha, \theta_{f}=\theta$. And when the reflection property of the object surface is assumed to be the diffuse reflection, the BRDF becomes

$$
\rho(\theta, \phi ; \theta, \phi)=\frac{r}{\pi} \cos \theta
$$

where $r$ is the reflectance ratio of the object surface. Then the following equation is obtained from eq. (5).

$$
z=-\frac{1}{2} \frac{D}{f} \sqrt{\frac{I r}{E_{C C D}^{F}-E_{C C D}^{N F}}} \cos ^{3} \alpha \cos ^{\frac{3}{2}} \theta
$$

Eq. (7) shows that the distance is proportional to the square root of the radiant intensity of the flash and the reflectance ratio of the object surface, and inverse-proportional to the square root of the difference of the irradiance of the object surface. In this stage, range information cannot be obtained because $\theta$ is unknown. A method to estimate $\theta$ is given in the next section.

\section{ESTIMATION OF THE NORMAL OF THE OBJECT SURFACE}

The reflected light becomes weak in proportion to cosine of the angle made by the visual axis and the normal of the object surface. So, it is necessary that the $\theta$ which is the angle made by the visual axis and the normal of the object surface is estimated.

In Fig. 2, $P_{i}$ and $P_{i+1}$ are the points of the object surface corresponding to the $i$-th and the $(i+1)$-th pixel of the CCD respectively. $\boldsymbol{\alpha}_{i}$ and $\boldsymbol{\alpha}_{i+1}$ are the vector from the center of the lens to the $i$-th pixel and $(i+1)$-th 
pixel of the CCD respectively. $E_{i}$ and $E_{i+1}$ are the irradiance only by the flash light obtained from the difference of two images. Let us assume the object surface is locally planar, and the normal is $\mathbf{n}$.

The vector $\overrightarrow{P_{i} P_{i+1}}$ is expressed as

$$
\overrightarrow{P_{i} P_{i+1}}=\overrightarrow{O P_{i+1}}-\overrightarrow{O P_{i}}=z_{i+1} \boldsymbol{\alpha}_{i+1}-z_{i} \boldsymbol{\alpha}_{i}
$$

Since $\overrightarrow{P_{i} P_{i+1}}$ is vertical to $\mathbf{n}$, the following equation is obtained.

$$
\mathbf{n} \cdot\left(z_{i+1} \boldsymbol{\alpha}_{i+1}-z_{i} \boldsymbol{\alpha}_{i}\right)=z_{i+1} \mathbf{n} \cdot \boldsymbol{\alpha}_{i+1}-z_{i} \cdot \boldsymbol{\alpha}_{i}=0
$$

From eq. (9), following equation is obtained.

$$
\frac{z_{i+1}}{z_{i}} \frac{\mathbf{n} \cdot \boldsymbol{\alpha}_{i+1}}{\mathbf{n} \cdot \boldsymbol{\alpha}_{i}}=1
$$

$\mathbf{n} \cdot \boldsymbol{\alpha}_{i}$ can be expressed as

$$
\mathbf{n} \cdot \boldsymbol{\alpha}_{i}=|\mathbf{n}|\left|\boldsymbol{\alpha}_{i}\right| \cos \theta_{i}
$$

Then eq. (10) is expressed as

$$
\frac{z_{i+1}}{z_{i}} \frac{\left|\boldsymbol{\alpha}_{i+1}\right|}{\left|\boldsymbol{\alpha}_{i}\right|} \frac{\cos \theta_{i+1}}{\cos \theta_{i}}=1
$$

From eq. (7),

$$
\frac{z_{i+1}}{z_{i}}=\left(\frac{E_{i}}{E_{i+1}} \frac{\cos ^{6} \alpha_{i+1}}{\cos ^{6} \alpha_{i}} \frac{\cos ^{3} \theta_{i+1}}{\cos ^{3} \theta_{i}}\right)^{\frac{1}{2}}
$$

From eq. (12) and (13), the following equation is obtained.

$$
\frac{z_{i+1}}{z_{i}}=\left(\frac{E_{i}}{E_{i+1}} \frac{\cos ^{6} \alpha_{i+1}}{\cos ^{6} \alpha_{i}} \frac{\left|\boldsymbol{\alpha}_{i}\right|^{3}}{\left|\boldsymbol{\alpha}_{i+1}\right|^{3}}\right)^{\frac{1}{5}}
$$

When the right side of eq. (14) is set to $a$, it can be expressed as follows.

$$
z_{i+1}=a z_{i}
$$

Using $a, \overrightarrow{P_{i} P_{i+1}}$ is expressed as

$$
\overrightarrow{P_{i} P_{i+1}}=z_{i}\left(\begin{array}{c}
a \tan \alpha_{i+1}-\tan \alpha_{i} \\
a-1
\end{array}\right)
$$

The direction of the vector parallel to the object surface can be obtained by eq. (16) using only known information.

Using the technique, direction of the vector parallel to the object surface can be obtained around the target pixel, and estimation of the normal of the object surface becomes possible. Then, $\theta$ is calculated by using the estimated normal of the object surface.

\section{ESTIMATION OF THE REFLECTANCE RATIO}

It is necessary to estimate the reflectance ratio of the object surface because the intensity of the reflected light depends also on the reflectance ratio of the object surface.

When it is assumed that the scene is irradiated almost evenly, the ratio of the irradiance of each pixel of the CCD without flash is equal to the ratio of the object surface. Therefore, the reflectance ratio for the whole scene can be estimated when there is the object whose reflectance ratio is known in the image.

We suppose that the reflectance ratio of the portion with the highest irradiance in the image without the flash is 0.9 , and then the whole reflectance ratio is estimated. 


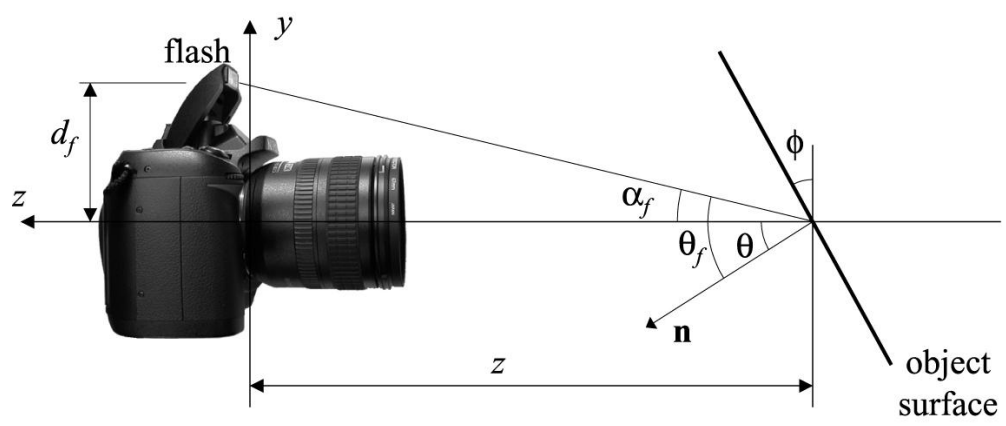

Figure 3. Influence of the assumption that the position of the flash is same as the position of the center of the lens

\section{ASSUMPTIONS IN THIS METHOD}

Assumptions in this method are as follows.

(a) The object surface has only diffuse reflection.

(b) The position of the flash is same as the position of the center of the lens.

(c) The brightest portion in the scene has the reflectance ratio of 0.9 .

The influence of (b) to the calculation result is described as follows.

In Fig. 3, the relation between the actual distance $z$ and the calculated distance $\hat{z}$ can be expressed with the following equation.

$$
\hat{z}=z \sqrt{\frac{\cos ^{3} \phi}{\cos ^{2}\left(\tan ^{-1}\left(\frac{d_{f}}{z}\right)\right) \cos ^{3}\left(\phi+\tan ^{-1}\left(\frac{d_{f}}{z}\right)\right)}}
$$

where $\phi$ is the angle of the inclination of the surface and $d_{f}$ is the distance of the flash and the center of the lens. Let us set $d_{f} 90 \mathrm{~mm}$ (the size of the camera used for experiments) and $z$ is $-1.0 \mathrm{~m}$. When $\phi$ is 0 degree, $\hat{z}$ is calculated as $-1.01 \mathrm{~m}$. When $\phi$ is 60 degrees, $\hat{z}$ is calculated as $-1.30 \mathrm{~m}$. It indicates that for large inclination angle, the approximation error becomes large.

\section{EXPERIMENTS}

We show experimental results using the proposed method. The normal was estimated using the technique described in Section 3 at each pixel.

The digital camera used for the experiments was Nikon D70, and its built-in flash was used. The lens was Nikon AF-S DX Zoom-Nikkor 18-70mm f/3.5 4.5G IF ED.

In the experiments, it was assumed that the flash is point light and the radiant intensity of the flash is equal in all directions.

\subsection{Plane with normal parallel to the optical axis}

A drawing paper $1.3 \mathrm{~m}$ away was measured. The result is shown in Fig. 4. It shows that the measurement values are distributed mostly on the plane of $1.3 \mathrm{~m}$, which is the correct value. The nonuniform characteristics of the lens and the CCD, etc. are expected as the cause of errors.

\subsection{Plane with normal not parallel to the optical axis}

The same object was measured from the direction which inclined 30 degrees from the optical axis. The distance on the optical axis is the same as that of Sec. 6.1.

The result is shown in Fig. 5(a), (b). The result calculated without estimating the normal, which was set to the parallel to the optical axis, is shown in Fig. 5(c). These figures show that the distance was calculated properly by estimation of the normal. 


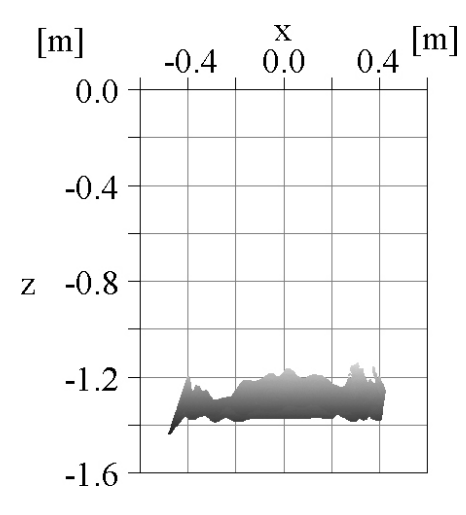

(a) Calculated distances

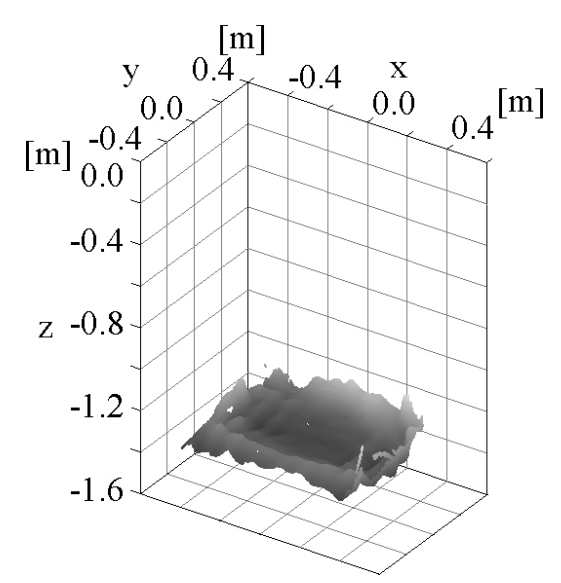

(b) Bird's-eye view

Figure 4. Measurement results of a plane with normal parallel to the optical axis

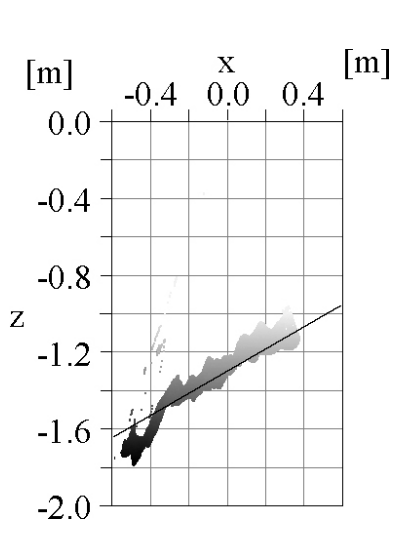

(a) Calculated distances

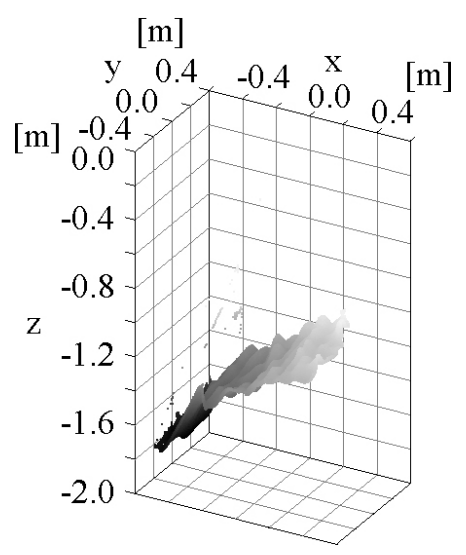

(b) Bird's-eye view

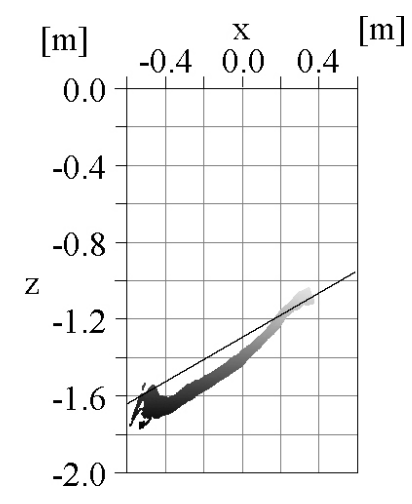

(c) Without estimation of normal

Figure 5. Measurement results of a plane with normal not parallel to the optical axis

\subsection{Various reflectance ratios in a scene}

The object used in this experiment is the Kodak Gray Scale(Fig. 6). It was measured from the front. The distance between the object and the camera was $1.2 \mathrm{~m}$. In this experiment, the normal was not estimated because the aim of this experiment was validation of estimation of the reflectance ratio. The normal was set parallel to the optical axis.

The actual and the estimated reflectance ratios are shown in Table 1. The result is shown in Fig. 7(a), (b). Fig. 7(c) shows one line's data on the gray scale. The result without estimation of the reflectance ratio, which was set constant to 0.9, is shown in Fig. 8(b). These results show that the reflectance ratio can be estimated well with the proposed method.

Table 1. Estimated reflectance ratios of a gray scale

\begin{tabular}{r|cccccccccccccc}
\hline actual & 0.89 & 0.79 & 0.63 & 0.50 & 0.40 & 0.32 & 0.25 & 0.20 & 0.16 & 0.13 & 0.10 & 0.08 & 0.06 & 0.05 \\
estimated & 0.90 & 0.67 & 0.51 & 0.40 & 0.31 & 0.23 & 0.19 & 0.16 & 0.11 & 0.09 & 0.07 & 0.06 & 0.05 & 0.04 \\
\hline
\end{tabular}




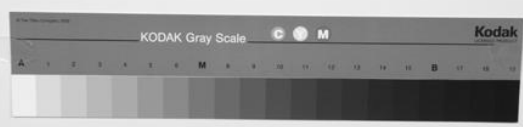

Figure 6. Kodak Gray Scale for the following experiments

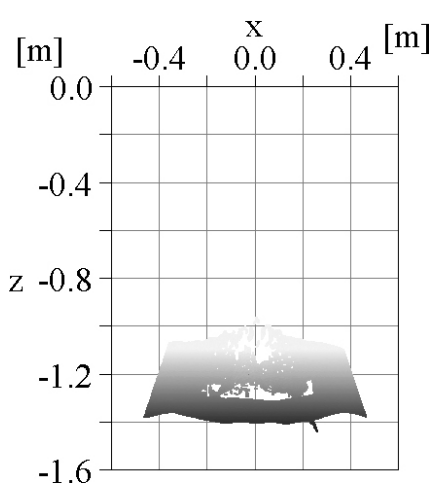

(a) Calculated distances

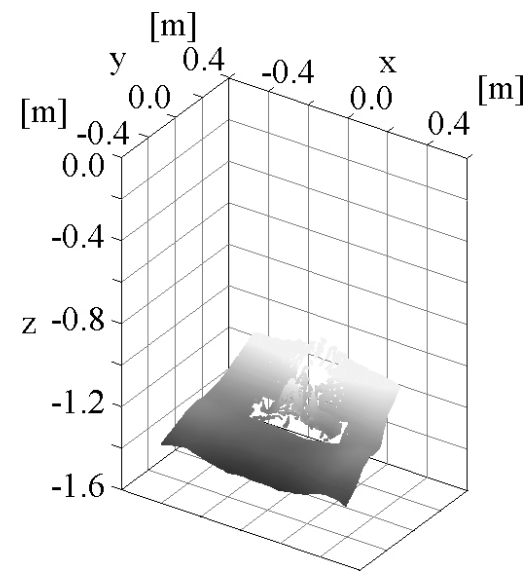

(b) Bird's-eye view

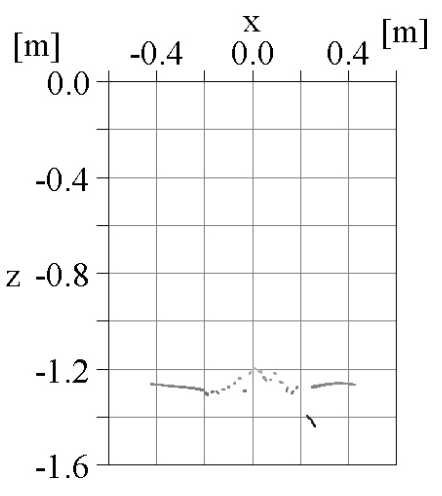

(c) One line's data on the gray scale

Figure 7. Measurement results of a gray scale (various reflectance ratios)

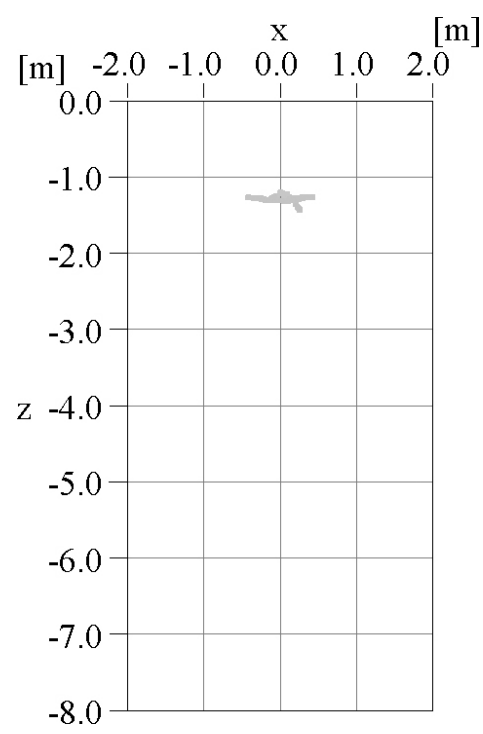

(a) With estimation of reflectance ratios

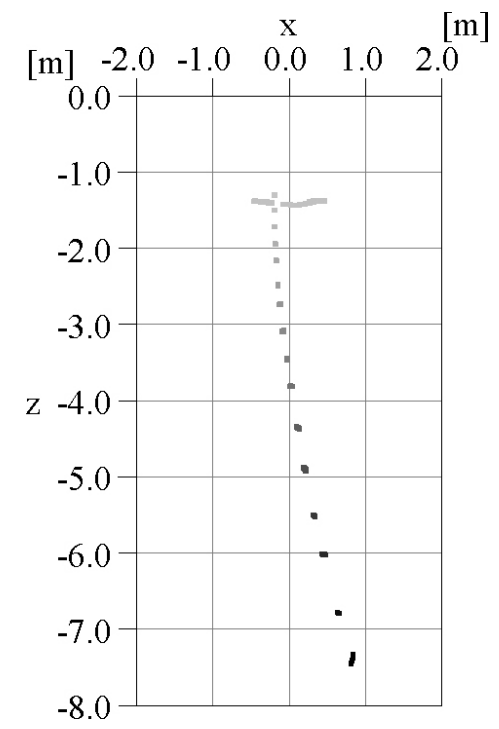

(b) Without estimation of reflectance ratios

Figure 8. Effect of estimation of reflectance ratios for a gray scale 


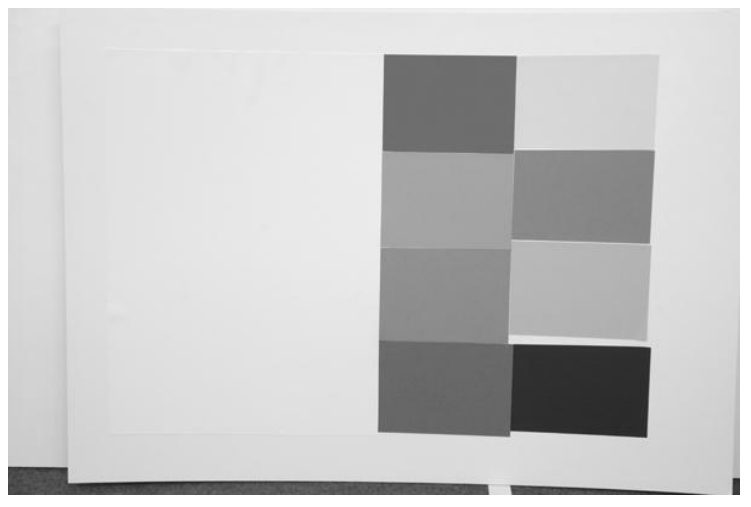

Figure 9. Colored paper for the following experiments

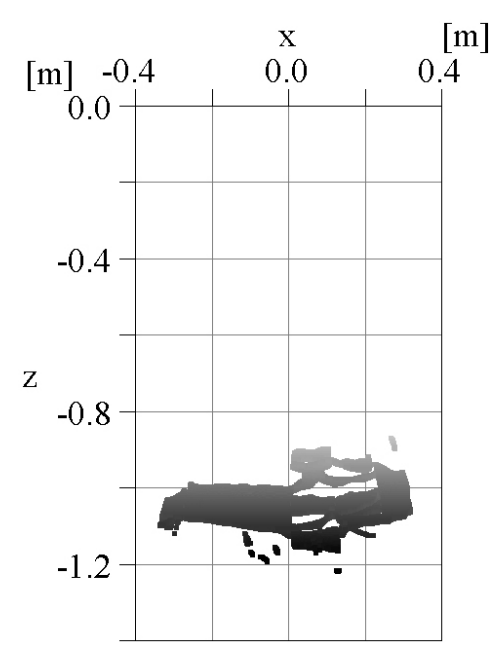

(a) Calculated distances

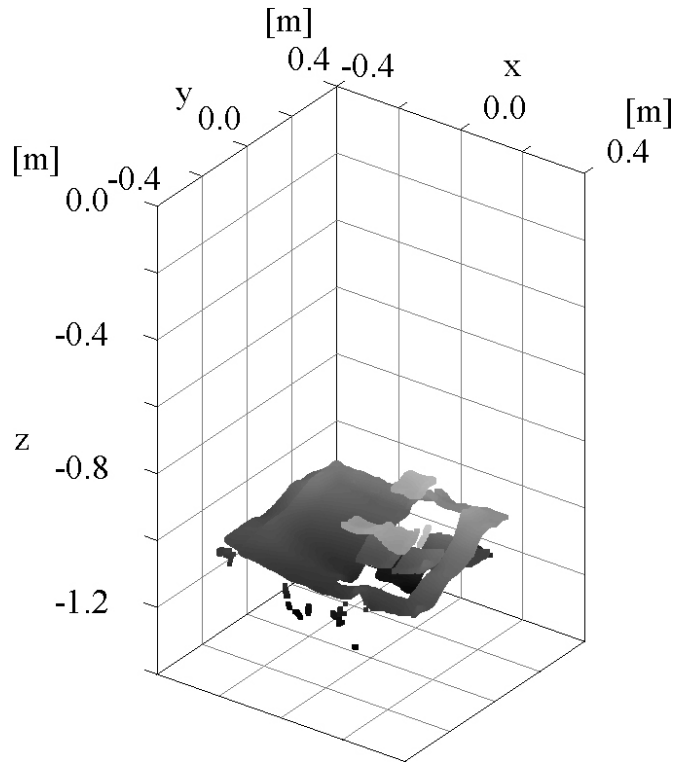

(b) Bird's-eye view

Figure 10. Measurement results of colored papers

\subsection{Colored paper}

A colored drawing paper(Fig. 9) was measured from the front. The normal was not estimated in this experiment either. The result is shown in Fig. 10. The result without estimation is shown in Fig. 11(b). These results show that the reflectance ratio is estimated well for the colored objects with the proposed method.

\section{CONCLUSION}

In this paper, we proposed a method to measure the distance by a digital camera using only the flash, without a large-scale equipment or a special environment. The method can cope with the normal and the reflectance ratio of an object. At the future work, it is necessary to apply the proposed method to the object with various shapes. 


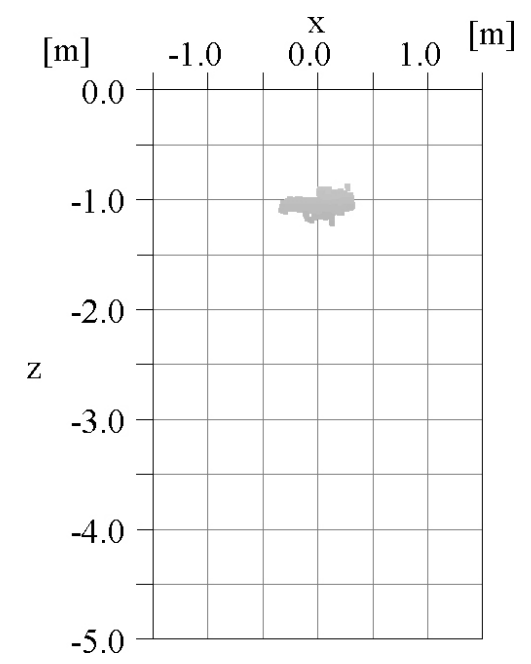

(a) With estimation of reflectance ratios

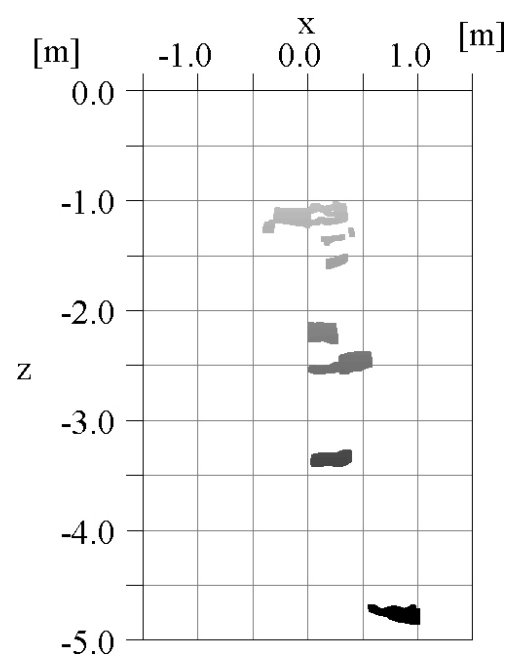

(b) Without estimation of reflectance ratios

Figure 11. Effect of estimation of reflectance ratios for colored papers

\section{REFERENCES}

1. R.A. Jarvis, "A perspective on range finding techniques for computer vision", Trans. PAMI-5, 2, pp.122-139, 1983.

2. P. Besl, "Active, Optical Range Imaging Sensors", Machine Vision and Applications, 1, 2, pp.127-152, 1988.

3. F. Blais, "A Review of 20 Years of Ranges Sensor Development", Videometrics VII, Proceedings of SPIE-IS\&T Electronic Imaging, SPIE Volume 5013, pp.62-76, 2003.

4. For example, Point Grey Research Inc. http://www.ptgrey.com/

5. M. Rioux, "Laser Range Finder Based on Synchronized Scanners", Applied Optics, 23, 21, pp.3837-3844, 1984.

6. B.K.P. Horn, Robot Vision, MIT Press, 1986.

7. K. Fujimura, et al., "Handheld camera 3D modeling system using multiple reference panels", Proc. of SPIE, Vol. 4661, pp.30-38, 2002.

8. S. Numazaki et al., "A kinetic and 3D image input device", Proc. of CHI'98, pp.237-238, 1998. 